\title{
Conhecimento e Aprendizado como impulsionadores da competitividade para a Indústria 4.0: análise bibliométrica
}

Versão do autor aceita publicada online: 28 mar. 2021

Publicado online: 30 jun. 2021

Como citar esse artigo - American Psychological Association (APA): Senna, D. A., \& Ribeiro, J. S. de A. N. (2021). Conhecimento e aprendizado como impulsionadores da competitividade para a Indústria 4.0: análise bibliométrica. Exacta. https://doi.org/10.5585/exactaep.2021.18916

\section{Diego Augustus Senna}

augustus.senna@yahoo.com.br

https://orcid.org/0000-0002-2765-726X

Universidade Federal de Minas Gerais (UFMG) e Pontifícia Universidade Católica de Minas Gerais (PUC Minas).

Bacharel em Engenharia Civil pela Universidade Federal de Minas Gerais - UFMG (2018). Mestrando em Saneamento, Meio Ambiente e Recursos Hídricos - UFMG (2019/2021). MBA em Indústria 4.0 pela Pontifícia Universidade Católica de Minas Gerais - PUC Minas (2020). Especializando em Inteligência Artificial e Aprendizado de Máquina - PUC Minas (2020/2021).

\section{Jurema Suely de Araújo Nery Ribeiro}

jurema.nery@gmail.com

https://orcid.org/0000-0002-6465-6020

Universidade FUMEC, Universidade UEMG e Universidade PUC Minas.

Doutora em Sistemas de Informação e Gestão do Conhecimento - FUMEC. Possui Mestrado em Administração - Área de pesquisa: Estratégia e Competitividade - pela Faculdade de Estudos Administrativos de Minas Gerias - FEAD (2008); MBA em Logística pelo Centro Universitário de Ciências Gerenciais - UNA (2004); MBA em Finanças pelo Centro Universitário de Ciências Gerenciais - UNA (2004); MBA em Gestão de Instituições pelo Núcleo de Pós Graduação Pitágoras (2011), Especialização em Administração da Produção pelo Instituto de Educação Tecnológica - IETEC (1997); Bacharel em Administração pelo Centro Universitário Newton Paiva (1991).

\section{Resumo}

A Indústria 4.0 é caracterizada por ampla utilização de tecnologias digitais e promove profundas alterações nas relações de produção e consumo. Há modificação do perfil da força de trabalho e, consequentemente, o sucesso competitivo depende do estudo e da compreensão da gestão do conhecimento e da aprendizagem. Este trabalho busca explorar, através de análises baseadas em revisão sistemática e bibliometria, como os construtos Indústria 4.0, Conhecimento e Aprendizado têm sido relacionados na literatura. Artigos foram identificados e categorizados considerando tipo de conhecimento estudado, metodologia, abordagem e países de origem dos autores. Identificou-se que predominam estudos associados à preparação acadêmica e à junção 
dos conhecimentos humano e de máquinas. Trabalhos teóricos com estudos de caso são os mais comuns, sendo aplicações práticas cada vez mais frequentes, principalmente no contexto acadêmico. Há predominância de países de economia mais desenvolvida nas publicações. $\mathrm{O}$ tema é ainda recente e esta pesquisa pode ser expandida.

Palavras-chave: Indústria 4.0. Conhecimento. Aprendizado. Bibliometria. Revisão Sistemática de Literatura.

\title{
Knowledge and Learning as drivers of competitiveness for Industry 4.0: a bibliometric analysis
}

\begin{abstract}
Industry 4.0 is characterized by widespread use of digital technologies and promotes deep changes in production and consumption relationships. The workforce profile is changing and, consequently, studying and understanding the management of knowledge and learning is essential for competitive success. This work seeks to explore, through systematic review and bibliometric analyses, how the constructs Industry 4.0, Knowledge and Learning are being related in literature. Articles were identified and categorized considering the type of knowledge studied, methodology, approach and countries of origin of authors. The results show that studies associated with academic preparation and the combination of human and machine knowledge are predominant. Theoretical works with case studies are the most common and practical applications are increasingly frequent, especially in the academic context. Countries with more developed economies are prevalent in publications. The topic is still recent and this research may be expanded.
\end{abstract}

Keywords: Industry 4.0. Knowledge. Learning. Bibliometrics. Systematic Literature Review. 


\section{Introdução}

A Quarta Revolução Industrial, também denominada Indústria 4.0 (I4.0), Manufatura Avançada ou Fábricas Inteligentes, caracteriza-se pela digitalização da produção, que possibilitou a personalização da fabricação em massa. Ao promover a utilização de tecnologias como Internet móvel, sensores menores e mais poderosos e Inteligência Artificial, introduziu profundas alterações nas relações de produção e consumo. Incentiva, continuamente, o desenvolvimento de novos modelos de negócios (Finance, 2015; Freire, Tosta, Helou Filho, \& Silva, 2017; Schwab, 2016). Há tendência de crescimento da colaboração entre os diferentes atores presentes ao longo das cadeias de suprimentos, algo que já ocorria anteriormente à I4.0, sendo as organizações tradicionais incentivadas a reconsiderar o notório distanciamento no relacionamento com seus fornecedores (Vanalle, 2011).

Através de procedimentos de coleta e análise de grande fluxo de dados provenientes de múltiplas fontes, a Indústria 4.0 possibilita que as organizações sejam capazes de fornecer respostas mais rápidas e flexíveis aos anseios do mercado, enquanto simultaneamente ganham em eficiência, qualidade e redução de custos. Esses componentes levarão, consequentemente, ao aumento da produtividade e do crescimento industrial. Considerando a colaboração cada vez maior entre seres humanos e máquinas, há inevitável modificação do perfil da força de trabalho. Justifica-se, portanto, a necessidade de acompanhar as rápidas mudanças para direcionar ações preventivas e corretivas.

Para gerenciar as novas competências, o conhecimento organizacional precisa se reinventar. Logo, compreende-se que o sucesso competitivo está relacionado ao estudo e à compreensão da gestão do conhecimento e da aprendizagem. Este trabalho busca, por meio de análises baseadas em revisão sistemática e bibliometria, explorar como o assunto tem sido abordado

na literatura. Foram considerados, nos trabalhos analisados, a composição autoral, o periódico, o ano de publicação, a metodologia, a abordagem, o tipo de conhecimento estudado e os países de origem dos autores - tomando como referência suas universidades.

Como objetivo geral, busca-se explorar como o relacionamento entre os construtos Indústria 4.0, Conhecimento e Aprendizado tem sido realizado em artigos científicos publicados em periódicos. Foram definidos quatro objetivos específicos: 1) investigar se existem autores frequentes e periódicos preferenciais; 2) analisar a distribuição das publicações conforme categorias definidas para o conhecimento; 3 ) identificar as principais metodologias adotadas e 
sua relação com as categorias e anos de publicação; e 4) e verificar a distribuição das publicações entre países.

O trabalho encontra-se organizado em cinco seções, incluída esta introdução. A seção 2 é composta pelo referencial teórico, que contempla os três construtos selecionados para a pesquisa. A metodologia é apresentada na seção 3, sendo os resultados demonstrados e discutidos na seção 4. Por fim, algumas considerações finais foram realizadas na seção 5.

\section{Referencial Teórico}

O referencial teórico encontra-se dividido em subseções referentes à Indústria 4.0 (2.1), ao conhecimento (2.2) e ao aprendizado (2.3).

\subsection{Indústria 4.0}

A “Plattform Industrie 4.0”, lançada, em 2013, como um conjunto de políticas públicas propostas pelo governo alemão, representa marco de ruptura com modelos tradicionais de produção e é marcada pelo incentivo à participação de máquinas no ambiente de trabalho, com fluxo crescente e ininterrupto de informações (Kagermann, Anderl, Gausemeier, Schuh, \& Wahlster, 2016). É natural que existam, portanto, questionamentos a respeito da possibilidade de declínio da importância do trabalhador humano ao longo do tempo. Davenport and Prusak (1998) e Teixeira Filho (2000), em contexto ainda distante da Indústria 4.0, consideravam que, no futuro, a verdadeira vantagem competitiva estaria na capacidade e na velocidade do aprendizado das pessoas nas organizações. Atualmente, autores como Schwab (2016) reforçam a colocação e ressaltam que o talento das forças de trabalho tende a ser cada vez mais importante que o capital, representando fator crítico e central para a produção.

Se as tecnologias são cada vez mais comuns, o conhecimento humano tende a ser diferencial mais evidente, essencial para o desenvolvimento de processos inteligentes (Stocker, Brandl, Michalczuk, \& Rosenberger, 2014). No contexto da Indústria 4.0 pode-se considerar, portanto, crescente tendência de democratização das tecnologias, havendo redução de custos para a aquisição de equipamentos antes considerados inacessíveis por diversas empresas. Logo, acentua-se, cada vez mais, a importância das competências dos colaboradores que irão interagir com esses sistemas. 
Considerando a exigência de conhecimentos técnicos e tecnológicos mais sofisticados e de nível superior, são cada vez mais aplicados conceitos como as redes para aprendizagem online e os programas de desenvolvimento humano para a inovação (geração de ideias, colaboração, compartilhamento e coprodução). Há avanço da gestão do conhecimento e do capital intelectual, ocorrendo o simultâneo desenvolvimento de novas competências e novas profissões (Aires, Kempner-Moreira, \& Freire, 2017). A ergonomia digital pode trazer benefícios como fluxo mais rápido de conhecimento, melhoria na experiência de trabalho e redução da complexidade das tarefas operativas.

Visando interpretar os impactos da Indústria 4.0 nas relações de trabalho e identificar categorias de estudo, é necessário compreender os pilares da Indústria 4.0, bem como seus construtos de suporte - conhecimento e aprendizado - apresentados na Figura 1

Figura 1 - Pilares e construtos de suporte da Indústria 4.0.

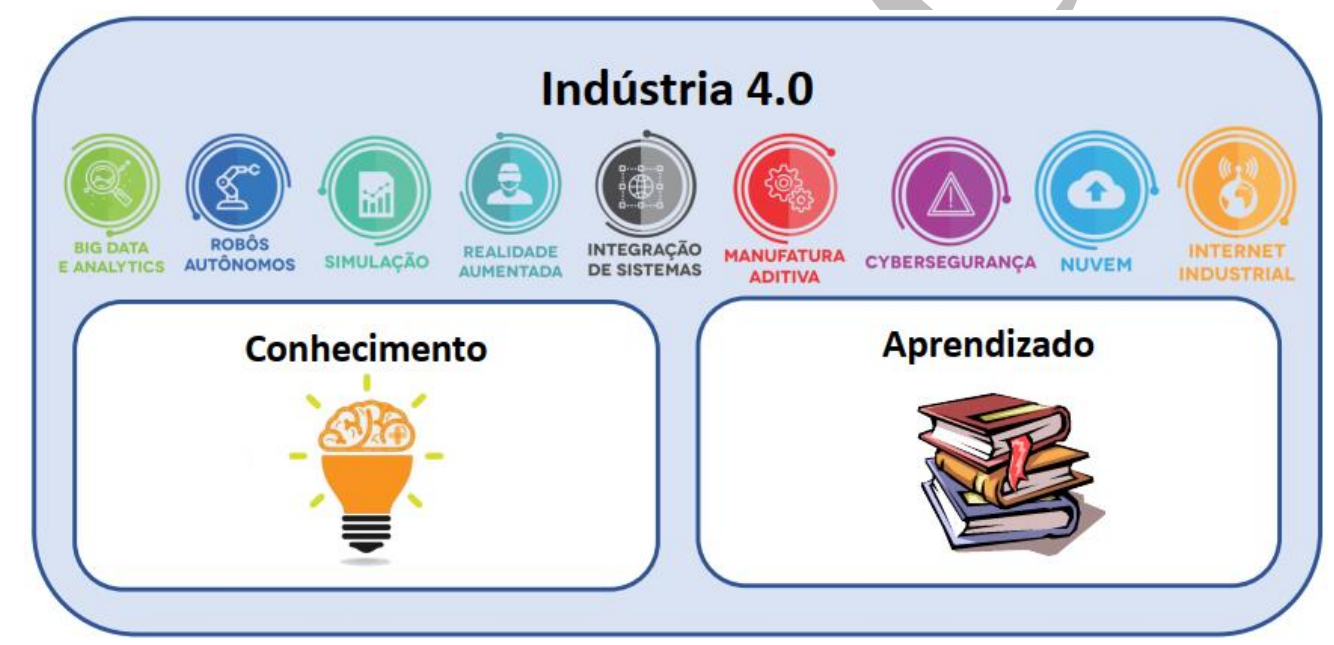

Fonte: Autores (2020).

Pode-se observar, a partir da análise da Figura 1, que as temáticas relacionadas à Quarta

Revolução Industrial dependem diretamente de conhecimentos específicos, sendo necessária a constante qualificação dos colaboradores, dada a velocidade das mudanças. A aplicação de técnicas de Big Data e simulação, por exemplo, é diretamente dependente de profissionais de estatística e programação que devem estudar o mercado para determinar o tipo de conhecimento a ser buscado, quais algoritmos podem ser aplicados e, principalmente, se os resultados apresentam significados relevantes para as organizações.

Robôs autônomos e a integração de sistemas, por sua vez, dependem de profundo acompanhamento das tecnologias e linguagens de programação mais recentes, vez que 
pequenas atualizações podem resultar em ganhos de eficiência e manutenção da vantagem competitiva. A cybersegurança, a utilização da nuvem e a Internet Industrial estão enquadradas no mesmo contexto, ocorrendo diferenciação pela questão da segurança, ponto de especial atenção para as empresas modernas e a sociedade como um todo. Por fim, a realidade aumentada e a Manufatura Aditiva estão diretamente relacionadas à transição de uma relação de cooperação para colaboração ativa entre funcionários e máquinas, constantemente conectados.

\subsection{Conhecimento}

A falta de consolidação de conhecimento adequado atuou sempre como uma das maiores barreiras para o sucesso das empresas. $\mathrm{Na}$ Indústria 4.0, torna-se elemento ainda mais crítico, vez que pode facilmente inviabilizar a implementação de tecnologias (Chong et al., 2018; Hariharasudan \& Kot, 2018). Estudos apontam a falta de conhecimento maduro, compreensivo e alinhado às tendências de mercado como a fonte mais comum de falhas na transformação digital (Ansari, Glawar, \& Nemeth, 2019; Küsters, Praß, \& Gloy, 2017).

O fluxo mais rápido de conhecimento, requerido pela Indústria 4.0, ocorre pela adoção da Gestão do Conhecimento (GC) e tem emergido como necessidade para todas as organizações que desejam melhorar seus resultados para serem mais competitivas, vez que o valor competitivo está neste ativo intangível tão disponível e, ao mesmo tempo, tão almejado (Dalkir, 2013; Nonaka, Von Krogh, \& Voelpel, 2006; Oliva, 2014; Ribeiro, 2019; Wiig, 1997). No contexto da Quarta Revolução Industrial, pode-se dizer que a gestão do conhecimento assume a "finalidade de criar um ambiente de aprendizagem contínuo para que a gestão das competências seja uma realidade. Isso não significa que para gerir competência é preciso gerir conhecimento, mas a gestão do conhecimento cria o ambiente psicológico propício para tal” (Ribeiro, Soares, Jurza, \& Ziviani, 2016, p. 10).

Considerando o fluxo acelerado de informações, havendo contato constante com outras pessoas, o trabalho em equipe é também fundamental na Indústria 4.0 e, consequentemente, o compartilhamento do conhecimento é ponto de grande relevância, vez que promove o desenvolvimento de habilidades do time. Dessa forma, o potencial de capital intelectual da organização alcança patamar ainda mais elevado e pode incluir não apenas funcionários internos, mas também diversos atores envolvidos ao longo da cadeia de suprimentos 
(Kaasinen et al., 2019; Kinkel, Schemmann, \& Lichtner, 2017; Stachová, Papula, Stacho, \& Kohnová, 2019).

\subsection{Aprendizado}

Com relação ao aprendizado, a temática pode ser analisada nas esferas humana, artificial ou na junção de ambas. Ressalta-se que, embora a participação de máquinas com Inteligência Artificial (IA) para tomar decisões próprias seja frequentemente associada à Indústria $4.0 \mathrm{e}$ representada na mídia, o trabalhador humano continua sendo o pilar de sustentação do ambiente produtivo, alcançando a máxima flexibilidade - que não pode ser obtida por máquinas - e dependendo de qualificação constante (Gorecky, Khamis, \& Mura, 2017).

A qualificação dos colaboradores será, na Quarta Revolução Industrial, cada vez mais voltada a habilidades de trabalho inovadoras, diferenciadas e específicas, direcionando sua atuação do campo laboral e físico para o campo intelectual (Barbieri, Ciabuschi, Fratocchi, \& Vignoli, 2017). Naturalmente que diversas dessas habilidades estarão associadas à junção entre o aprendizado humano e o de máquinas. Embora o desenvolvimento tecnológico, ligado à produção gerenciada por computadores, seja muito importante, seu máximo potencial pode ser alcançado apenas por meio da interação com trabalhadores humanos.

O pensamento da aprendizagem organizacional desenvolveu-se ao longo do tempo e muitas perspectivas surgiram. Chiva e Alegre (2005) identificaram duas perspectivas: individual e social. Chiva-Gómez (2004) explica que a perspectiva individual considera a aprendizagem como fenômeno individual e, consequentemente, entende que as organizações aprendem através de indivíduos que aprendem (Senge, 1990). A perspectiva social, no entanto, considera a aprendizagem como fenômeno social e, como tal, entende que as organizações aprendem através de comunidades e grupos (Lave \& Wenger, 1991).

Os avanços na área mostram que as organizações e as pessoas nelas inseridas precisam aprender constantemente, facilitando a obtenção de conhecimento para todos os membros da empresa, os quais, por sua vez, transformam continuamente a companhia através de seus serviços, produtos e inovações que emergem do processo de aprendizagem (Kumpikaite, 2008). 


\section{Metodologia}

Nos meses de junho e julho de 2019, realizou-se pesquisa para localizar artigos científicos publicados em periódicos (articles) que relacionassem a Indústria 4.0, o conhecimento e o aprendizado, sem restrições de ano de publicação ou idioma, seguindo os protocolos dispostos no Quadro 1. A metodologia de análise foi baseada em revisão sistemática de literatura e bibliometria, coletando dados sobre as publicações e analisando-os de forma qualiquantitativa através de critérios pré-definidos (Martínez-Silveira, Silva, \& Laguardia, 2014).

Quadro 1 - Protocolos de pesquisa

\begin{tabular}{|c|l|}
\hline Protocolo & \multicolumn{1}{c|}{ Descrição } \\
\hline $\begin{array}{c}\text { Quadro } \\
\text { conceitual }\end{array}$ & $\begin{array}{l}\text { Gerenciamento e atualização do conhecimento e do aprendizado, nos meios industrial e } \\
\text { acadêmico, considerando o contexto da Indústria 4.0 e a interface humano-máquina. }\end{array}$ \\
\hline Justificativa & $\begin{array}{l}\text { A sobrevivência de empresas e universidades depende de sua capacidade de atualização de } \\
\text { conhecimentos e cultura para operar na Indústria 4.0. Objetiva-se explorar como a literatura } \\
\text { tem abordado o gerenciamento de conhecimento e meios de aprendizado, tendo em vista a } \\
\text { rápida evolução tecnológica. }\end{array}$ \\
\hline $\begin{array}{c}\text { Descritores } \\
\text { pesquisados }\end{array}$ & $\begin{array}{l}\text { Considerando título, palavras-chave ou resumo, a busca foi restrita às seguintes expressões: } \\
\text { "Industry 4.0" AND "Learning" AND "Knowledge". }\end{array}$ \\
\hline Bases de dados & Ebsco, Scielo, Scopus, Spell. \\
\hline $\begin{array}{c}\text { Critérios de } \\
\text { exclusão }\end{array}$ & Repetição ou falta de aderência à temática. \\
\hline
\end{tabular}

Fonte: Autores (2020).

Apenas as plataformas Scopus e Scielo retornaram resultados. Na plataforma Scopus, foram encontrados 39 estudos, sendo um desconsiderado por falta de aderência ao tema. A plataforma Scielo retornou apenas um resultado, descartado por estar também presente na amostra encontrada na Scopus. Os 38 documentos selecionados foram analisados em planilhas no software LibreOffice Calc. Para cada artigo foram extraídos o título, as palavraschave, os autores, o ano de publicação, os países de origem das universidades do autor principal e dos coautores, o periódico, a abordagem - quantitativa, qualitativa ou qualiquantitativa - e a metodologia. Os países de origem foram, então, classificados em duas categorias: países do Norte, de economia mais desenvolvida, e países do Sul, de economia em desenvolvimento.

Utilizando-se revisão sistemática, uma das quatro categorias dispostas no Quadro 2 foi atribuída a cada trabalho com base na principal temática estudada. Os pilares da Indústria 4.0, apresentados na Figura 1, justificam a divisão da gestão do conhecimento nessas categorias. Alguns estudos são direcionados apenas à parcela técnica, ao tratar de assuntos como algoritmos para aplicação em Inteligência Artificial e manutenção preditiva automática, 
formando a categoria Aprendizado de Máquina. Outros tratam apenas do aprendizado e da consolidação de conhecimento humano dentro das empresas, por exemplo, ao tratar de assuntos psicológicos e novas técnicas de gamificação e serious games, e constituem a categoria Aprendizado Humano.

Quadro 2 - Categorias consideradas

\begin{tabular}{|c|l|}
\hline Categoria & \multicolumn{1}{|c|}{ Descrição } \\
\hline $\begin{array}{c}\text { Aprendizado } \\
\text { Humano }\end{array}$ & $\begin{array}{l}\text { Artigos com foco no gerenciamento de conhecimento e modelos de aprendizagem para } \\
\text { os recursos humanos no ambiente de trabalho. }\end{array}$ \\
\hline $\begin{array}{c}\text { Aprendizado de } \\
\text { Máquina }\end{array}$ & $\begin{array}{l}\text { Estudos voltados ao aprendizado de máquina, abrangendo tecnologias e sistemas } \\
\text { relacionados à automatização e ao conhecimento não humano no ambiente de trabalho. }\end{array}$ \\
\hline $\begin{array}{c}\text { Aprendizado } \\
\text { Humano-Máquina }\end{array}$ & $\begin{array}{l}\text { Pesquisas que discutem tanto o conhecimento humano quanto o de máquina, } \\
\text { especificando relações entre ambos no ambiente de trabalho. }\end{array}$ \\
\hline $\begin{array}{c}\text { Preparação } \\
\text { Acadêmica }\end{array}$ & $\begin{array}{l}\text { Artigos voltados ao sistema educacional ou ambiente de pesquisa, principalmente } \\
\text { universitário, especificando inovações relacionadas ao contexto da Indústria 4.0. }\end{array}$ \\
\hline
\end{tabular}

Fonte: Autores (2020).

Considerando a crescente dependência entre os mundos real e virtual, é natural que ambas as temáticas sejam trabalhadas em conjunto em inúmeros estudos, formando a categoria de Aprendizado Humano-Máquina, que contém artigos que tratam de assuntos como a implementação de tecnologias de realidade aumentada e os impactos nas atividades laborais ao operar os equipamentos. Por fim, considerando a grande demanda por aprendizado diferenciado e atualizado, o setor educacional, abrangendo escolas, cursos técnicos e profissionalizantes e universidades, constitui a categoria de Preparação Acadêmica, que trata de assuntos como a utilização de fábricas de aprendizado como meio de treinamento para futuros profissionais.

Análises foram realizadas a partir dos dados coletados para que fossem identificadas tendências, construindo embasamento para a realização de inferências qualitativas. Primeiramente, a composição autoral foi avaliada para determinar se haveria repetição frequente de algum autor, e o número de publicações entre periódicos foi investigado buscando encontrar preferências. A distribuição das publicações entre as quatro categorias foi, então, analisada, sendo posteriormente correlacionada com o fator temporal - ano de publicação - e metodologias e abordagens.

Por fim, a distribuição das publicações entre países foi verificada considerando primeiramente apenas o local da universidade do autor principal - como referência de origem do estudo - e, em seguida, as universidades de todos os autores. Um documento contendo a listagem dos 
países associados a cada autor ou coautor foi elaborado e utilizado como texto-base para a construção de uma nuvem de palavras no software Wordle.

\section{Resultados}

Os 38 trabalhos da amostra estão apresentados no Quadro 3.

Quadro 3 - Identificação dos trabalhos encontrados

\begin{tabular}{|c|c|c|c|}
\hline $\mathbf{N}^{\mathbf{0}}$ & Ano & Autor & Título \\
\hline 1 & 2014 & $\begin{array}{l}\text { Stocker, A., Brandl, P., Michalczuk, } \\
\text { R., \& Rosenberger, M. }\end{array}$ & Human-centred ICT tools for smart factories \\
\hline 2 & 2016 & Synnes, E. L., \& Welo, T. & $\begin{array}{l}\text { Bridging the Gap between High and Low-volume Pro- } \\
\text { duction through Enhancement of Integrative Capabili- } \\
\text { ties }\end{array}$ \\
\hline 3 & 2017 & $\begin{array}{l}\text { Baena, F., Guarin, A., Mora, J., Sauza, } \\
\text { J., \& Retat, S. }\end{array}$ & Learning Factory: The Path to Industry 4.0 \\
\hline 4 & 2017 & $\begin{array}{l}\text { ElMaraghy, H., Moussa, M., ElMara- } \\
\text { ghy, W., \& Abbas, M. }\end{array}$ & $\begin{array}{l}\text { Integrated Product / System Design and Planning for } \\
\text { New Product Family in a Changeable Learning Factory }\end{array}$ \\
\hline 5 & 2017 & Gorecky, D., Khamis, M., \& Mura, K. & $\begin{array}{l}\text { Introduction and establishment of virtual training in the } \\
\text { factory of the future }\end{array}$ \\
\hline 6 & 2017 & $\begin{array}{l}\text { Hold, P., Erol, S., Reisinger, G., \& } \\
\text { Sihn, W. }\end{array}$ & Planning and Evaluation of Digital Assistance Systems \\
\hline 7 & 2017 & Kamp, B., Ochoa, A., \& Di & $\begin{array}{l}\text { Smart servitization within the context of industrial user- } \\
\text { supplier relationships: contingencies according to a ma- } \\
\text { chine tool manufacturer }\end{array}$ \\
\hline 8 & 2017 & $\begin{array}{l}\text { Karre, H., Hammer, M., Kleindienst, } \\
\text { M., \& Ramsauer, C. }\end{array}$ & $\begin{array}{l}\text { vards an Industry } 4.0 \text { State of the LeanLab } \\
\text { arsity of Technology }\end{array}$ \\
\hline 9 & 2017 & $\begin{array}{l}\text { Kinkel, S., Schemmann, B., \& Licht- } \\
\text { ner, R. }\end{array}$ & $\begin{array}{l}\text { Critical Competencies for the Innovativeness of Value } \\
\text { Creation Champions: Identifying Challenges and Work- } \\
\text { integrated Solutions }\end{array}$ \\
\hline 10 & 2017 & Küsters, D., Praß, N., \& Gl & $\begin{array}{l}\text { Textile Learning Factory } 4.0 \text { - Preparing Germany's } \\
\text { Textile Industry for the Digital Future }\end{array}$ \\
\hline 11 & 2017 & & $\begin{array}{l}\text { The AAU Smart Production Laboratory for Teaching } \\
\text { and Research in Emerging Digital Manufacturing Tech- } \\
\text { nologies }\end{array}$ \\
\hline 12 & 2017 & $\begin{array}{l}\text { Reuter, M., Oberc, H., Wannöffel, M., } \\
\text { Kreimeier, D., Klippert, J., Pawlicki, } \\
\text { P., \& Kuhlenkötter, B. }\end{array}$ & $\begin{array}{l}\text { Learning Factories' Trainings as an Enabler of Proactive } \\
\text { Workers' Participation Regarding Industry } 4.0\end{array}$ \\
\hline 13 & 2017 & Shamim, S., Cang, S., Yu, H., \& Li, Y. & $\begin{array}{l}\text { Examining the feasibilities of Industry } 4.0 \text { for the hospi- } \\
\text { tality sector with the lens of management practice }\end{array}$ \\
\hline 14 & 2017 & $\begin{array}{l}\text { Thalmann, S., Pammer-Schindler, V., } \\
\text { Fessl, A., \& Weghofer, F. }\end{array}$ & $\begin{array}{l}\text { Learning 4.0: Addressing challenges for employees suc- } \\
\text { cessfully }\end{array}$ \\
\hline 15 & 2017 & $\begin{array}{l}\text { Uhlemann, T. H.-J., Schock, C., Leh- } \\
\text { mann, C., Freiberger, S., \& Steinhilper, } \\
\text { R. }\end{array}$ & $\begin{array}{l}\text { The Digital Twin: Demonstrating the Potential of Real } \\
\text { Time Data Acquisition in Production Systems }\end{array}$ \\
\hline 16 & 2017 & $\begin{array}{l}\text { Vila, C., Ugarte, D., Ríos, J., \& } \\
\text { Abellán, J. V. }\end{array}$ & $\begin{array}{l}\text { oject-based collaborative engineering learning to de- } \\
\text { lop Industry } 4.0 \text { skills within a PLM framework }\end{array}$ \\
\hline 17 & 2017 & $\begin{array}{l}\text { Yakovle, V. V., Khasanov, M. M., Sit- } \\
\text { nikov, A. N., Prokofiev, D. O., Pus- } \\
\text { tovskikh, A. A., Margarit, A. S., Simo- } \\
\text { nov, M. V., \& Perets, D. S. }\end{array}$ & $\begin{array}{l}\text { The direction of cognitive technologies development in } \\
\text { the Upstream Division of Gazprom Neft Company }\end{array}$ \\
\hline 18 & 2017 & $\begin{array}{l}\text { Zeidler, F., Bayhan, H., Venkatapathy, } \\
\text { A. K. R., \& Hompel, M. T. }\end{array}$ & $\begin{array}{l}\text { Reference field for research and development of novel } \\
\text { hybrid forms of human machine interaction in logistics }\end{array}$ \\
\hline 19 & 2018 & $\begin{array}{l}\text { Ansari, F., Khobreh, M., Seidenberg, } \\
\text { U., \& Sihn, W. }\end{array}$ & $\begin{array}{l}\text { A problem-solving ontology for human-centered cyber } \\
\text { physical production systems }\end{array}$ \\
\hline
\end{tabular}




\begin{tabular}{|c|c|c|c|}
\hline 20 & 2018 & $\begin{array}{l}\text { Barbieri, P., Ciabuschi, F., Fratocchi, } \\
\text { L., \& Vignoli, M. }\end{array}$ & What do we know about manufacturing reshoring? \\
\hline 21 & 2018 & $\begin{array}{l}\text { Chong, S., Pan, G.-T., Chin, J., Show, } \\
\text { P. L., Yang, T. C. K., \& Huang, C.-M. }\end{array}$ & $\begin{array}{l}\text { Integration of 3D Printing and Industry } 4.0 \text { into Engi- } \\
\text { neering Teaching }\end{array}$ \\
\hline 22 & 2018 & $\begin{array}{l}\text { Gitelman, L. D., \& Kozhevnikov, M. } \\
\text { V. }\end{array}$ & $\begin{array}{l}\text { Paradigm of Managerial Education for a Technological } \\
\text { Breakthrough in the Economy }\end{array}$ \\
\hline 23 & 2018 & $\begin{array}{l}\text { Guo, Q., Miyamae, Y., Wang, Z., Ta- } \\
\text { niuchi, K., Yang, H., \& Liu, Y. }\end{array}$ & $\begin{array}{l}\text { Senvis-Net: Learning from imbalanced machinery data } \\
\text { by transferring visual element detectors }\end{array}$ \\
\hline 24 & 2018 & Hariharasudan, A., \& Kot, S. & $\begin{array}{l}\text { A Scoping Review on Digital English and Education } 4.0 \\
\text { for Industry } 4.0\end{array}$ \\
\hline 25 & 2018 & enz, J., Wuest, T., \& Westkämper, E. & Holistic approach to machine tool data analytics \\
\hline 26 & 2018 & $\begin{array}{l}\text { Morozov, D., Lezoche, M., \& Panetto, } \\
\text { H. }\end{array}$ & Multi-paradigm modelling of Cyber-Physical Systems \\
\hline 27 & 2018 & $\begin{array}{l}\text { Sá Carvalho, E. S. de, \& Duarte Filho, } \\
\text { N. F. }\end{array}$ & $\begin{array}{l}\text { Proposal for a mobile learning system focusing on the } \\
\text { characteristics and applications practical of industry } 4.0\end{array}$ \\
\hline 28 & 2019 & Ansari, F., Glawar, R., \& Nemeth, T. & $\begin{array}{l}\text { PriMa: a prescriptive maintenance model for cyber- } \\
\text { physical production systems }\end{array}$ \\
\hline 29 & 2019 & Chou, P.-N., \& Feng, S.-T. & $\begin{array}{l}\text { Using a tablet computer application to advance high } \\
\text { school students' laboratory learning experiences: A fo- } \\
\text { cus on electrical engineering education }\end{array}$ \\
\hline 30 & 2019 & $\begin{array}{l}\text { Diez-Olivan, A., Del Ser, J., Galar, D., } \\
\text { \& Sierra, B. }\end{array}$ & $\begin{array}{l}\text { Data fusion and machine learning for industrial progno- } \\
\text { sis: Trends and perspectives towards Industry } 4.0\end{array}$ \\
\hline 31 & 2019 & Ghani, E. K., \& Muhammad, K. & $\begin{array}{l}\text { Industry 4.0: Employers' expectations of accounting } \\
\text { graduates and its implications on teaching and learning } \\
\text { practices }\end{array}$ \\
\hline 32 & 2019 & $\begin{array}{l}\text { Kaasinen, E., Schmalfuß, F., Özturk, } \\
\text { C., Aromaa, S., Boubekeur, M., Hei- } \\
\text { lala, J., Heikkilä, P., Kuula, T., Liina- } \\
\text { suo, M., Mach, S., Mehta, R., Petäjä, } \\
\text { E., \& Walter, T. }\end{array}$ & $\begin{array}{l}\text { Empowering and engaging industrial workers with Op- } \\
\text { erator } 4.0 \text { solutions }\end{array}$ \\
\hline 33 & 2019 & Nicoletti, L., \& Padovano, & $\begin{array}{l}\text { Emergency preparedness in industrial plants: A forward- } \\
\text { looking solution based on industry } 4.0 \text { enabling technol- } \\
\text { ogies }\end{array}$ \\
\hline 34 & 2019 & Lu, Y., Xie, R. & $\begin{array}{l}\text { Bearing fault diagnosis with nonlinear adaptive diction- } \\
\text { ary learning }\end{array}$ \\
\hline 35 & 2019 & $\begin{array}{l}\text { Stachová, K., Papula, J., Stacho, Z., \& } \\
\text { Kohnová, L. }\end{array}$ & $\begin{array}{l}\text { External partnerships in employee education and devel- } \\
\text { opment as the key to facing industry } 4.0 \text { challenges }\end{array}$ \\
\hline 36 & 2019 & $\begin{array}{l}\text { Siddoo, V., Sawattawee, J., Janchai, } \\
\text { W., \& Thinnukool, O. }\end{array}$ & $\begin{array}{l}\text { An exploratory study of digital workforce competency } \\
\text { in Thailand }\end{array}$ \\
\hline 37 & 2019 & & $\begin{array}{l}\text { Industry } 4.0 \text { and capability development in manufactur- } \\
\text { ing subsidiaries }\end{array}$ \\
\hline 38 & 2019 & Tavcar, J., \& Horvath, I. & $\begin{array}{l}\text { A review of the principles of designing smart cyber- } \\
\text { physical systems for run-time adaptation: Learned les- } \\
\text { sons and open issues }\end{array}$ \\
\hline
\end{tabular}

Fonte: Autores (2020).

Conforme apresentado no Quadro 3, apenas dois autores estiveram presentes em mais de um artigo: Ansari, F. atuou como autor principal em dois trabalhos (artigos 19 e 28), enquanto Sihn, W. foi coautor de também dois trabalhos (artigos 6 e 19). Conclui-se, portanto, que não há autores frequentes considerando os documentos da amostra. Com relação aos periódicos, apenas o Procedia Manufacturing (11 artigos, ou 28,9\%), o Sustainability - Switzerland (3 artigos, ou 7,9\%) e o International Journal of Computer Integrated Manufacturing (2 artigos, 
ou 5,3\%) estiveram associados a mais de uma publicação. Há, portanto, destaque para o primeiro, que parece atrair maior interesse. Nenhum dos trabalhos da amostra foi publicado em periódico brasileiro.

A distribuição de publicações por categorias, considerando as temáticas indicadas no Quadro 2, está disposta na Tabela 1. Os dados foram organizados em função das metodologias e abordagens empregadas nos trabalhos.

Tabela 1 - Distribuição de publicações por categoria, abordagem e metodologia

\begin{tabular}{|c|c|c|c|}
\hline Metodologias & P. Acadêmica & A. Humano-Máquina & A. Humano \\
\hline Teórico com estudo de caso & $8(47,1 \%)$ & $3(42,9 \%)$ & 0 \\
\hline Teórico & $3(17,6 \%)$ & $3(42,9 \%)$ & $2(50,0 \%)$ \\
\hline Teórico com pesquisa qualitativa & 0 & $1(10,0 \%)$ & 0 \\
\hline Pesquisa qualitativa & $3(17,6 \%)$ & $2(20,0 \%)$ & $1(25,0 \%)$ \\
\hline Bibliometria & $2(11,8 \%)$ & $1(10,0 \%)$ & 0 \\
\hline Estudo de caso & $1(5,9 \%)$ & $1(14,3 \%)$ & $1(25,0 \%)$ \\
\hline Abordagens & P. Acadêmica & A. Humano-Máquina & A. Humano \\
\hline Qualitativa & $9(52,9 \%)$ & $8(80,0 \%)$ & $3(75,0 \%)$ \\
\hline Quantitativa & 0 & $1(10,0 \%)$ & 0 \\
\hline Qualiquantitativa & $8(47,1 \%)$ & $1(10,0 \%)$ & $1(25,0 \%)$ \\
\hline Total & $17(44,8 \%)$ & $10(26,3 \%)$ & $4(10,5 \%)$ \\
\hline
\end{tabular}

Fonte: Autores (2020).

A análise da Tabela 1 indica que publicações voltadas ao setor educacional, com ênfase na preparação acadêmica para o mercado de trabalho, são dominantes, representadas por 17 artigos $(44,8 \%)$, o que demonstra a grande importância do tema. O resultado aponta para a forte movimentação da academia, que busca incluir atividades compatíveis com novas competências (Barbieri et al., 2017). No setor industrial, predominam artigos que abordam o aprendizado humano-máquina, com 10 trabalhos (26,3\%), o que reforça a crescente interdependência entre o conhecimento humano e o não humano. Juntas, as duas categorias correspondem a 71,1\% do total. As amostras referentes às outras duas categorias são reduzidas e admitiu-se que não apresentariam caráter representativo.

A Tabela 2 apresenta a distribuição de publicações por ano, conforme a metodologia utilizada. 
Tabela 2 - Distribuição das publicações por ano, conforme a metodologia utilizada

\begin{tabular}{l|c|c|c|c|c|c|c|c}
\hline \multicolumn{1}{c|}{ Metodologia } & $\mathbf{2 0 1 4}$ & $\mathbf{2 0 1 6}$ & $\mathbf{2 0 1 7}$ & $\mathbf{2 0 1 8}$ & $\mathbf{2 0 1 9}$ & Total & \% & \% Ac. \\
\hline Teórico com estudo de caso & - & - & 7 & 3 & 4 & 14 & 36,8 & 36,8 \\
\hline Teórico & 1 & - & 6 & 3 & 1 & 11 & 29,0 & 65,8 \\
\hline Teórico com pesquisa qualitativa & - & - & 1 & - & - & 1 & 2,6 & 68,4 \\
\hline Pesquisa qualitativa & - & - & - & 1 & 5 & 6 & 15,8 & 84,2 \\
\hline Estudo de caso & - & 1 & 2 & - & - & 3 & 7,9 & 92,1 \\
\hline Bibliometria & - & - & - & 2 & 1 & 3 & 7,9 & 100,0 \\
\hline Total & 1 & 1 & 16 & 9 & 11 & 38 & 100,0 & 100,0 \\
\hline
\end{tabular}

Fonte: Autores (2020).

Com relação às metodologias mais frequentes, as Tabelas 1 e 2 indicam que artigos com conteúdo teórico e estudos de caso - que propõem e testam algum tipo de modelo relacionado à Indústria 4.0 - são os mais frequentes, contemplando 14 trabalhos $(36,8 \%)$. Do total da amostra, 11 documentos (29,0\%) são unicamente teóricos, e 6 (15,8\%) envolvem apenas pesquisa qualitativa. As demais metodologias aparecem em menos de $8 \%$ dos artigos.

Do total, 24 trabalhos $(63,1 \%)$ envolvem algum tipo de aplicação prática, seja estudo de caso ou pesquisa qualitativa, sendo os 14 restantes $(36,9 \%)$ apenas teóricos ou baseados em bibliometria. Com relação ao tipo de abordagem, 24 trabalhos $(63,1 \%)$ apresentam caráter qualitativo, enquanto $11(29,0 \%)$ são qualiquantitativos e apenas $3(7,9 \%)$ são exclusivamente quantitativos.

Considerando os 17 artigos referentes à preparação acadêmica, 12 (70,6\%) estão associados a aplicações práticas e $8(47,1 \%)$ apresentam caráter qualiquantitativo. Dos 10 trabalhos voltados ao aprendizado humano-máquina, relativos principalmente ao setor manufatureiro, 6 $(60,0 \%)$ apresentam teor prático e apenas $2(20,0 \%)$ adotam variáveis quantitativas na análise, sendo um puramente quantitativo e outro qualiquantitativo. No contexto do setor acadêmico, é expressiva a ocorrência de trabalhos práticos e com algum caráter quantitativo, o que pode indicar acentuado interesse pela rápida aplicação do conhecimento desenvolvido e por obtenção de feedback.

No cenário científico, temáticas de estudo recentes estão geralmente associadas a pesquisas teóricas, com pouca representatividade de aplicações práticas, pois algum tempo é necessário para a consolidação de novos conhecimentos. Por esse motivo, abordagens qualitativas tendem a ser também predominantes, vez que há poucos dados disponíveis para que sejam realizadas análises quantitativas. No caso da Indústria 4.0, que foi definida formalmente apenas em 2013 (Kagermann et al., 2016), a pressão exercida pela competitividade crescente 
e o próprio desenvolvimento tecnológico aceleram o processo de implementação de novas técnicas, o que poderia explicar o predomínio de estudos práticos na amostra (Finance, 2015; Freire et al., 2017; Schwab, 2016). Contudo, é importante ressaltar que grande parcela desses estudos está também associada a alguma inovação proposta, ou seja, ainda em caráter experimental.

O destaque da formulação de modelos - 26 artigos, ou 68,4\% da amostra, contêm modelagens teóricas - é relacionado à contemporaneidade do assunto, pois há amplo espaço para descobertas, e investimentos realizados por países em centros de pesquisa tendem a potencializar a elaboração de novas aplicações como forma de ganhar vantagem competitiva internacional.

Apenas dois artigos foram publicados antes de 2017, sendo o trabalho mais antigo referente ao ano de 2014 - o que condiz com a definição formal do termo Indústria $4.0 \mathrm{em} \mathrm{2013.} \mathrm{Silva,}$ Kovaleski e Pagani (2020) realizaram estudo bibliométrico buscando determinar a influência dos conceitos e tecnologias da I4.0 no ambiente industrial, sendo encontrada forte tendência de crescimento no ano de 2017 - havendo expansão após 2015 -, o que reforça os resultados obtidos neste trabalho.

Em 2017, 6 dos trabalhos publicados eram unicamente teóricos. Nos anos seguintes, mesmo ao considerar que foram incluídos, para o ano de 2019, apenas trabalhos publicados até o mês de julho, parece haver tendência de queda, enquanto metodologias como a pesquisa qualitativa e a bibliometria passaram a ser utilizadas e apresentam tendência de crescimento. O desenvolvimento de mais aplicações práticas promove a utilização de técnicas como estudos de caso e pesquisas qualitativas, enquanto o aumento do número de publicações possibilita que análises bibliométricas, inicialmente pouco viáveis, sejam realizadas.

A relação de países associados às publicações está apresentada na Tabela 3. 
Tabela 3 - Distribuição das publicações conforme países de origem

\begin{tabular}{l|c|c|c}
\hline \multicolumn{1}{c|c}{ Países } & $\begin{array}{c}\text { Número de } \\
\text { publicações }\end{array}$ & Porcentagem & $\begin{array}{c}\text { Porcentagem } \\
\text { Acumulada }\end{array}$ \\
\hline Alemanha & 7 & $18,4 \%$ & $18,4 \%$ \\
\hline Áustria & 6 & $15,8 \%$ & $34,2 \%$ \\
\hline Espanha & 3 & $7,9 \%$ & $42,1 \%$ \\
\hline Itália, Malásia e Rússia & 2 & $5,3 \%$ & $58,0 \%$ \\
\hline $\begin{array}{l}\text { Brasil, Canadá, China, Colômbia, Dinamarca, } \\
\text { Eslováquia, Eslovênia, Estados Unidos, Finlândia, }\end{array}$ & 1 & $2,6 \%$ & $100,0 \%$ \\
$\begin{array}{l}\text { França, Hungria, Índia, Noruega, Reino Unido, } \\
\text { Tailândia e Taiwan }\end{array}$ & & & \\
\hline
\end{tabular}

Fonte: Autores (2020).

Identifica-se, a partir da análise da Tabela 3, que seis países concentram quase $60 \%$ das publicações, com destaque para a Alemanha, associada a 7 trabalhos $(18,4 \%)$, a Áustría, relacionada a $6(15,8 \%)$, e a Espanha, vinculada a $3(7,9 \%)$. Dentre os demais países, apenas Itália, Malásia e Rússia contam com mais de uma publicação. Os dados indicam que a grande maioria dos trabalhos está associada às maiores economias do mundo, países do Norte, sendo a representatividade de países do Sul, como o Brasil, muito pequena. Entre os países do Norte, destaca-se a presença de nações europeias. Comprova-se, portanto, que há concentração de publicações, o que indica grande distância de competitividade industrial entre países do Norte e países do Sul.

Estiveram envolvidas, nos trabalhos, 145 pessoas. Destas, $127(87,6 \%)$ estão associadas a universidades instaladas em países do Norte, enquanto apenas 18 (12,4\%) têm relação com instituições de países do Sul. A nuvem de palavras - construída a partir das universidades de referência de todos os autores de forma a evidenciar diferenças na participação - está apresentada na Figura 2.

Figura 2 - Nuvem de palavras dos países de origem das universidades de todos os autores

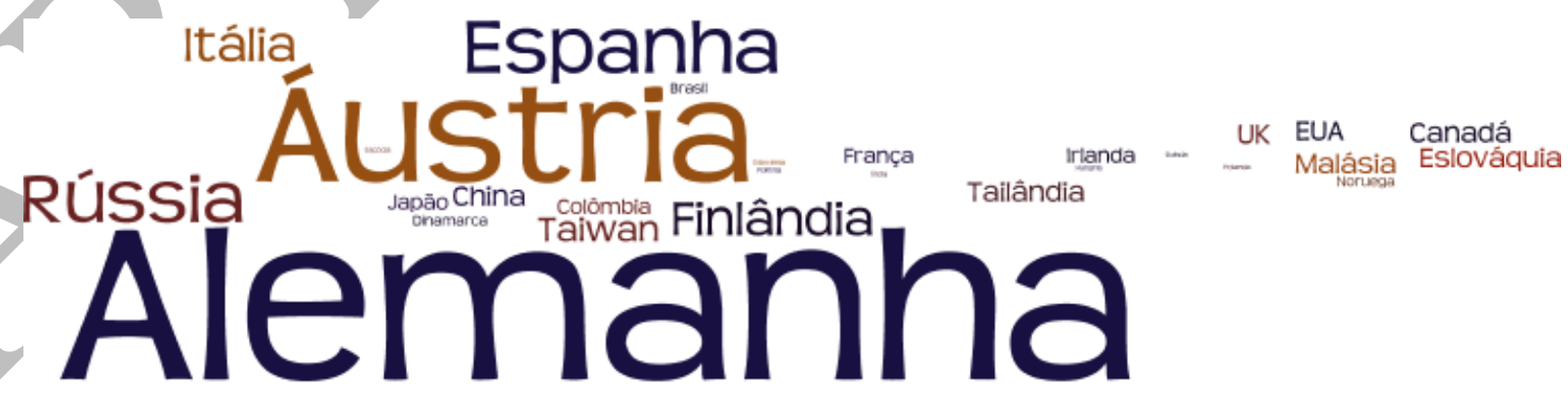

Fonte: Autores (2020).

O conceito da Indústria 4.0 surgiu na Alemanha e é esperado que o País tenha destaque, principalmente ao considerar os investimentos feitos pelo governo em centros de pesquisa, o 
que se confirma ao analisar a Figura 2, pois $32(22,1 \%)$ dos 145 autores estão associados a universidades alemãs. O mesmo cenário se aplica à Áustria, que está geograficamente próxima da Alemanha e compartilha características culturais e socioeconômicas: 20 autores $(13,8 \%)$ estão relacionados a instituições de ensino austríacas. Considerando os outros países destacados anteriormente, 11 autores (7,6\%) são vinculados a universidades espanholas, 10 $(6,9 \%)$ a universidades russas, $7(4,8 \%)$ a universidades italianas e $4(2,8 \%)$ a universidades malaias.

A baixa representatividade de países como Estados Unidos e China pode estar relacionada ao uso de diferentes termos, sendo menos comum a adoção da expressão "Indústria 4.0", o que dificulta a localização de pesquisas utilizando-se os construtos deste trabalho. Nos Estados Unidos, por exemplo, são utilizados termos menos específicos, como Advanced Manufacturing e Industrial Internet (Arbix, Salerno, Zancul, Amaral, \& Lins, 2017; DiezOlivan, Del Ser, Galar, \& Sierra, 2018). Países como Estados Unidos e China apresentam, há algum tempo, notável presença em estudos associados à inteligência competitiva e campos relacionados, conforme demonstrado por Azarias, Coutinho e Cámpos (2018), e espera-se, portanto, que estejam fomentando pesquisas para melhorar sua competitividade industrial no contexto da I4.0.

Há inclinação ao aumento do número de pesquisas relacionadas ao assunto, embora a concentração em torno de países com economias mais desenvolvidas seja tendência de difícil alteração a curto prazo. O distanciamento tende a aumentar com a consolidação de redes de pesquisa dedicadas em países do Norte. Cabe aos governos de países do Sul, assim como às empresas sediadas nesses locais, a difícil tarefa de concentrar esforços em torno do investimento em universidades e centros de pesquisa, visando evitar que a distância em relação aos países do Norte seja cada vez mais ampliada. Diversas organizações e seus colaboradores sequer conhecem a Indústria 4.0: a falta de escolaridade e a preparação dos empregadores e empregados podem ser apontadas como barreiras críticas para seu desenvolvimento no Brasil (Pacchini, Santos, Logiudice, \& Lucato, 2020).

\section{Considerações Finais}

Conclui-se que os objetivos do estudo foram atingidos, vez que que os resultados decorrentes da análise dos dados permitiram realizar inferências relevantes para compreender como a temática do conhecimento e do aprendizado, no contexto da Indústria 4.0, tem sido abordada 
na literatura. De forma geral, conclui-se que o relacionamento entre os construtos é recente e ainda há muito a ser descoberto, sendo a intensificação da competitividade e o grande fluxo de dados responsáveis pelo rápido desenvolvimento de pesquisas.

O primeiro objetivo foi alcançado ao determinar que não há autores frequentes na amostra analisada, conforme apresentado no Quadro 3, embora exista um periódico dominante. $\mathrm{O}$ segundo foi verificado ao apontar, com base na Tabela 1, que predominam estudos associados à preparação acadêmica e ao aprendizado humano-máquina. $\mathrm{O}$ terceiro foi atingido ao identificar, por meio de análise das Tabelas 1 e 2, que predominam trabalhos teóricos com estudos de caso, unicamente teóricos ou baseados em pesquisas qualitativas, sendo estudos práticos cada vez mais frequentes, principalmente no contexto da academia. Por fim, o quarto e último objetivo foi averiguado, com base na Tabela 3 e na Figura 2, confirmando que existe clara predominância de autores associados a países do Norte nas publicações.

As limitações associadas ao estudo estão relacionadas à própria contemporaneidade do tema, tendo em vista que o pequeno número de artigos publicados dificulta a realização de inferências com maior grau de representatividade. Além disso, a pesquisa pode ser expandida considerando outras bases de dados científicas e variações regionais e linguísticas do construto Indústria 4.0.

\section{Referências}

Aires, R. W. do A., Kempner-Moreira, F. K., \& Freire, P. de S. (2017, setembro). Indústria 4.0: competências requeridas aos profissionais da quarta revolução industrial. Anais do Congresso Internacional de Conhecimento e Inovação (CIKI), Foz do Iguaçu, PR, Brasil, 7. Recuperado em 20 de junho, 2019, de: https://proceeding.ciki.ufsc.br/index.php/ciki/article/view/314

Ansari, F., Glawar, R., \& Nemeth, T. (2019). PriMa: a prescriptive maintenance model for cyber-physical production systems. International Journal of Computer Integrated Manufacturing, 32(4-5), 482-503. https://doi.org/10.1080/0951192X.2019.1571236

Ansari, F., Khobreh, M., Seidenberg, U., \& Sihn, W. (2018). A problem-solving ontology for human-centered cyber physical production systems. CIRP Journal of Manufacturing Science and Technology, 22, 91-106. https://doi.org/10.1016/j.cirpj.2018.06.002

Arbix, G., Salerno, M. S., Zancul, E., Amaral, G., \& Lins, L. M. (2017). Advanced Manufacturing: What Is to Be Learnt from Germany, the U.S., and China [O Brasil e a nova onda de manufatura avançada]. Novos Estudos, 36(3), 29-49. https://doi.org/10.25091/s0101$\underline{3300201700030003}$ 
Azarias, J. G., Coutinho, A. dos R., \& Campos, F. C. (2018). Análise bibliométrica sobre estudo de caso e Survey voltado a inteligência competitiva na indústria. Exacta, 16(4), 135 148. https://doi.org/10.5585/exactaep.v16n4.8331

Baena, F., Guarin, A., Mora, J., Sauza, \& J., Retat, S. (2017). Learning Factory: The Path to Industry 4.0. Procedia Manufacturing, 9, 73-80. https://doi.org/10.1016/j.promfg.2017.04.022

Barbieri, P., Ciabuschi, F., Fratocchi, L., \& Vignoli, M. (2018). What do we know about manufacturing reshoring? Journal of Global Operations and Strategic Sourcing, 11(1), 79122. https://doi.org/10.1108/JGOSS-02-2017-0004

Chiva, R, \& Alegre, J. (2005). Aprendizagem organizacional e conhecimento organizacional: para a integração de duas abordagens. Aprendizagem em gestão, 36(1), 49-68. https://doi.org/10.1177/1350507605049906

Chiva-Gómez, R. (2004). The facilitating factors for organizational learning in the ceramic sector. Human Resource Development International, 7(2), 233-249.

https://doi.org/10.1080/1367886042000243817

Chong, S., Pan, G.-T., Chin, J., Show, P.L., Yang, T.C.K., \& Huang, C.-M. (2018). Integration of 3D printing and industry 4.0 into engineering teaching. Sustainability (Switzerland), 10(11). https://doi.org/10.3390/su10113960

Chou, P.-N., \& Feng, S.-T. (2019). Using a tablet computer application to advance high school students' laboratory learning experiences: A focus on electrical engineering education.

Sustainability (Switzerland), 11(2). https://doi.org/10.3390/su11020381

Dalkir, K. (2013). Knowledge management in theory and practice. Londres: Routledge.

Davenport, T. H., \& Prusak, L. (1998). Conhecimento empresarial: como as empresas gerenciam o seu capital intelectual. Rio de Janeiro: Campus.

Diez-Olivan, A., Del Ser, J., Galar, D., \& Sierra, B. (2018). Data fusion and machine learning for industrial prognosis: Trends and perspectives towards Industry 4.0. Information Fusion, 50, 92-111. https://doi.org/10.1016/j.inffus.2018.10.005

ElMaraghy, H., Moussa, M., ElMaraghy, W., \& Abbas, M. (2017). Integrated Product / System Design and Planning for New Product Family in a Changeable Learning Factory. Procedia Manufacturing, 9, 65-72. https://doi.org/10.1016/j.promfg.2017.04.008

Finance, Audit Tax Consulting Corporate. (2015). Industry 4.0 Challenges and solutions for the digital transformation and use of exponential technologies. Finance, Audit Tax Consulting Corporate: Zurich, Swiss, 2015. Recuperado em 8 de agosto, 2019, de https://bit.ly/33ujbI3.

Freire, P. de S., Tosta, K. C. B. T., Helou Filho, E. A., \& Silva, G. G. da. (2012). Memória organizacional e seu papel na gestão do conhecimento. Revista de Ciências da Administração, 14(33), 41-51. http://dx.doi.org/10.5007/2175-8077.2012v14n33p41

Ghani, E. K., \& Muhammad, K. (2019). Industry 4.0: Employers' expectations of accounting graduates and its implications on teaching and learning practices. International Journal of Education and Practice, 7(1), 19-29. https://doi.org/10.18488/journal.61.2019.71.19.2918 
Gitelman, L. D., \& Kozhevnikov, M. V. (2018). Paradigm of Managerial Education for a Technological Breakthrough in the Economy. Economy of Region, 14(2), 433-449. https://doi.org/10.17059/2018-2-8

Gorecky, D., Khamis, M., \& Mura, K. (2017). Introduction and establishment of virtual training in the factory of the future. International Journal of Computer Integrated Manufacturing, 30(1), 182-190. https://doi.org/10.1080/0951192X.2015.1067918

Guo, Q., Miyamae, Y., Wang, Z., Taniuchi, K., Yang, H., \& Liu, Y. (2018). Senvis-Net: Learning from imbalanced machinery data by transferring visual element detectors. International Journal of Machine Learning and Computing, 8(5), 416-422. https://doi.org/10.18178/ijmlc.2018.8.5.722

Hariharasudan, A., \& Kot, S. (2018). A scoping review on Digital English and Education 4.0 for Industry 4.0. Social Sciences, 7(11). https://doi.org/10.3390/socsci7110227

Hold, P., Erol, S., Reisinger, G., \& Sihn, W. (2017). Planning and Evaluation of Digital Assistance Systems. Procedia Manufacturing, 9, 143-150. https://doi.org/10.1016/j.promfg.2017.04.024

Kaasinen, E., Schmalfuß, F., Özturk, C., Aromaa, S., Boubekeur, M., Heilala, J., Heikkilä, P., Kuula, T., Liinasuo, M., Mach, S., Mehta, R., Petäjä, E., \& Walter, T. (2019). Empowering and engaging industrial workers with Operator 4.0 solutions. Computers and Industrial Engineering. https://doi.org/10.1016/j.cie.2019.01.052

Kagermann, H., Anderl, R., Gausemeier, J., Schuh, G., \& Wahlster, W. (Eds.). (2016). Industrie 4.0 in a Global Context: Strategies for Cooperating with International Partners (acatech STUDY). Munique: Herbert Utz Verlag. Recuperado em 20 de junho, 2019, de: https://bit.ly/374TWjw

Kamp, B., Ochoa, A., \& Diaz, J. (2017). Smart servitization within the context of industrial user-supplier relationships: contingencies according to a machine tool manufacturer. International Journal on Interactive Design and Manufacturing, 11(3), 651-663. https://doi.org/10.1007/s12008-016-0345-0

Karre, H., Hammer, M., Kleindienst, M., \& Ramsauer, C. (2017). Transition towards an Industry 4.0: State of the LeanLab at Graz University of Technology. Procedia Manufacturing, 9, 206-213. https://doi.org/10.1016/i.promfg.2017.04.006

Kinkel, S., Schemmann, B., \& Lichtner, R. (2017). Critical Competencies for the Innovativeness of Value Creation Champions: Identifying Challenges and Work-integrated Solutions. Procedia Manufacturing, 9, 323-330. https://doi.org/10.1016/j.promfg.2017.04.021

Kumpikaite, V. (2008). Human resource development in learning organization. Journal of business economics and management, 9(1), 25-31. https://doi.org/10.3846/1611$\underline{1699.2008 .9 .25-31}$

Küsters, D., Praß, N., \& Gloy, Y.-S. (2017). Textile Learning Factory 4.0 - Preparing Germany's Textile Industry for the Digital Future. Procedia Manufacturing, 9, 214-221. https://doi.org/10.1016/j.promfg.2017.04.035 
Lave, J., \& Wenger, E. (1991). Situated learning: Legitimate peripheral participation. Cambridge University Press.

Lenz, J., Wuest, T., \& Westkämper, E. (2018). Holistic approach to machine tool data analytics. Journal of Manufacturing Systems, 48, 180-191.

https://doi.org/10.1016/j.jmsy.2018.03.003

Longo, F., Nicoletti, L., \& Padovano, A. (2019). Emergency preparedness in industrial plants: A forward-looking solution based on industry 4.0 enabling technologies. Computers in Industry, 105, 99-122. https://doi.org/10.1016/j.compind.2018.12.003

Lu, Y., Xie, R., \& Liang, S. Y. (2019). Bearing fault diagnosis with nonlinear adaptive dictionary learning. International Journal of Advanced Manufacturing Technology. https://doi.org/10.1007/s00170-019-03455-1

Madsen, O., \& Møller, C. (2017). The AAU Smart Production Laboratory for Teaching and Research in Emerging Digital Manufacturing Technologies. Procedia Manufacturing, 9, 106112. https://doi.org/10.1016/j.promfg.2017.04.036

Martinez-Silveira, M. S., Silva, C. H. da, Laguardia, J. (2014, outubro). A revisão sistemática como método em estudo bibliométrico. Anais do Encontro Nacional de Pesquisa em Ciência da Informação (ENANCIB), Belo Horizonte, MG, Brazil, 15. Recuperado em 20 de junho, 2019, de: https://www.arca.fiocruz.br/handle/icict/29527

Morozov, D., Lezoche, M., \& Panetto, H. (2018). Multi-paradigm modelling of CyberPhysical Systems. IFAC-PapersOnLine, 51(11), 1385-1390.

https://doi.org/10.1016/j.ifacol.2018.08.334

Nonaka, I., Von Krogh, G., \& Voelpel, S. (2006). Organizational knowledge creation theory: Evolutionary paths and future advances. Organization studies, 27(8), 1179-1208.

https://doi.org/10.1177/0170840606066312

Oliva, F. L. (2014). Knowledge management barriers, practices and maturity model. Journal of Knowledge Management, 18(6), 1053-1074. https://doi.org/10.1108/JKM-03-2014-0080

Pacchini, A. P. T., Santos, J. C. da S., Logiudice, R., \& Lucato, W. C. (2020). Indústria 4.0: barreiras para implantação na indústria brasileira. Exacta, 18(2), 278-292. https://doi.org/10.5585/ExactaEP.v18n2.10605

Reuter, M., Oberc, H., Wannöffel, M., Kreimeier, D., Klippert, J., Pawlicki, P., \& Kuhlenkötter, B. (2017). Learning Factories' Trainings as an Enabler of Proactive Workers' Participation Regarding Industrie 4.0. Procedia Manufacturing, 9, 354-360.

https://doi.org/10.1016/j.promfg.2017.04.020

Ribeiro, J. S. de A. N., Soares, M. A. C., Jurza, P., \& Ziviani, F. (2016, novembro). A articulação entre competências e inovação ancorada pela gestão do conhecimento visando a vantagem competitiva sustentável. Anais do Simpósio Internacional de Gestão de Projetos, Inovação e Sustentabilidade (SINGEP), São Paulo, SP, Brazil, 5. Recuperado em 20 de junho, 2019, de: https://bit.ly/37PyC0B

Ribeiro, J. S. de A. N. (2019). Modelo de análise do compartilhamento de conhecimento e inovação no desempenho de entrega: um estudo sobre Integração da Cadeia de Suprimentos. 
Tese. Tese de doutorado, Programa de Pós-Graduação em Sistemas de Informação e Gestão do conhecimento da Universidade FUMEC, Belo Horizonte, MG, Brasil.

Sá Carvalho, E. S. de, \& Duarte Filho, N. F. (2018). Proposta de um sistema de aprendizagem móvel com foco nas características e aplicações práticas da indústria 4.0. RISTI - Revista Ibérica de Sistemas e Tecnologias de Informação, 27, 36-51.

http://dx.doi.org/10.17013/risti.27.36-51

Schwab, K. (2016). A Quarta Revolução Industrial. São Paulo: Edipro.

Senge, P. (1990). The fifth discipline. Doubleday. Senge, PM (1992). Mental Models. Planning Review, 20(2), 4-13. New York: Currency.

Shamim, S., Cang, S., Yu, H., \& Li, Y. (2017). Examining the feasibilities of Industry 4.0 for the hospitality sector with the lens of management practice. Energies, 10 (4).

https://doi.org/10.3390/en10040499

Siddoo, V., Sawattawee, J., Janchai, W., \& Thinnukool, O. (2019). An exploratory study of digital workforce competency in Thailand. Heliyon, 5(5). https://doi.org/10.1016/j.heliyon.2019.e01723

Silva, V. L. da, Kovaleski, J. L., \& Pagani, R. N. (2020). Influências do conceito e das tecnologias da Indústria 4.0 no ambiente industrial. Exacta, 18(2), 420-437. https://doi.org/10.5585/ExactaEP.v18n2

Stachová, K., Papula, J., Stacho, Z., \& Kohnová, L. (2019). External partnerships in employee education and development as the key to facing industry 4.0 challenges. Sustainability (Switzerland), 11(2). https://doi.org/10.3390/su11020345

Stocker, A., Brandl, P., Michalczuk, R., \& Rosenberger, M. (2014). Human-centred ICT tools for smart factories. Elektrotechnik und Informationstechnik, 131(7), 207-211.

https://doi.org/10.1007/s00502-014-0215-z

Szalavetz, A. (2019). Industry 4.0 and capability development in manufacturing subsidiaries. Technological Forecasting and Social Change, 145, 384-395.

https://doi.org/10.1016/j.techfore.2018.06.027

Synnes, E. L., \& Welo, T. (2016). Bridging the Gap between High and Low-volume Production through Enhancement of Integrative Capabilities. Procedia Manufacturing, 5, 2640. https://doi.org/10.1016/j.promfg.2016.08.006

Tavcar, J., \& Horvath, I. (2019). A review of the principles of designing smart cyber-physical systems for run-time adaptation: Learned lessons and open issues. IEEE Transactions on Systems, Man, and Cybernetics: Systems, 49(1), 145-158.

https://doi.org/10.1109/TSMC.2018.2814539

Teixeira Filho, J. (2000). Gerenciando conhecimento: como a empresa pode usar a memória organizacional e a inteligência competitiva no desenvolvimento dos negócios. Senac.

Thalmann, S., Pammer-Schindler, V., Fessl, A., \& Weghofer, F. (2017). Learning 4.0: Addressing challenges for employees successfully. Productivity Management, 22(2), 62-64. 
Uhlemann, T. H.-J., Schock, C., Lehmann, C., Freiberger, S., \& Steinhilper, R. (2017). The Digital Twin: Demonstrating the Potential of Real Time Data Acquisition in Production Systems. Procedia Manufacturing, 9, 113-120. https://doi.org/10.1016/j.promfg.2017.04.043

Vanalle, R. M. (2011). Relacionamento cliente-fornecedor: evidências de estudos sobre a indústria automobilística. Exacta, 9(1), 13-28. https://doi.org/10.5585/Exacta.v9i1.2187

Vila, C., Ugarte, D., Ríos, J., \& Abellán, J. V. (2017). Project-based collaborative engineering learning to develop Industry 4.0 skills within a PLM framework. Procedia Manufacturing, 13, 1269-1276. https://doi.org/10.1016/j.promfg.2017.09.050

Yakovle, V. V., Khasanov, M. M., Sitnikov, A. N., Prokofiev, D. O., Pustovskikh, A. A., Margarit, A. S., Simonov, M. V., \& Perets, D. S. (2017). The direction of cognitive technologies development in the Upstream Division of Gazprom Neft Company. Neftyanoe Khozyaystvo - Oil Industry, 12, 6-9.

Wiig, K. M. (1997). Knowledge management: an introduction and perspective. Journal of knowledge Management, 1(1), 6-14. https://doi.org/10.1108/13673279710800682

Zeidler, F., Bayhan, H., Venkatapathy, A. K. R., \& Hompel, M. T. (2017). Reference field for research and development of novel hybrid forms of human machine interaction in logistics. Logistics Journal. https://doi.org/10.2195/lj_Proc_zeidler_de_201710_01 